\title{
PARTICIPACIÓN Y LIDERAZGO DE LAS MUJERES GUANAJUATENSES
}

Lucía Rodríguez Guzmán ${ }^{[1]}$ M. Guadalupe de los Auxilios Díaz Cisneros ${ }^{[2]}$

\section{Resumen}

Este estudio descriptivo y exploratorio, ha identificado en un grupo de mujeres las características de liderazgo, oportunidades, dificultades y propuestas desde la perspectiva de género. Se aplicaron cuestionarios a 55 mujeres del municipio de León, Guanajuato. Resultados: Algunas características de las líderes muestran: iniciativa, seguridad, compromiso, honestidad, liderazgo, conocimiento, carácter, actitud emprendedora, responsabilidad y sensibilidad, poder de decisión, cooperación, fortaleza, confianza, solidaridad, autonomía, servicio y búsqueda del bien común. Las oportunidades de participación en la vida pública tienen mayor apertura; sin embargo, todavía faltan programas de género inclusivo. En relación a las dificultades que enfrentan para proyectar sus ideas de cambio han mencionado como principales obstáculos el machismo, discriminación y falta de oportunidades educativas; el rol de ama de casa, la falta de programas de desarrollo, lucha por los ideales femeninos, libertad de expresión, falta de iniciativa y credibilidad, opresión, violencia y maltrato. Las propuestas para mejorar las opciones de desarrollo tienen como principales planteamientos: capacitación, educación, empoderamiento, empleo, oportunidades, apoyos gubernamentales, leyes justas, reconocimiento de capacidades, respeto, trabajo en equipo y desarrollo humano. La información es relevante para caracterizar los casos exitosos de mujeres que han logrado trascender hacia puestos de liderazgo dentro de una sociedad típicamente conservadora al interior del contexto mexicano.

Palabras clave: Mujeres; liderazgo; oportunidades de participación; contexto mexicano.

\section{Summary}

This descriptive and exploratory study has identified in a group of women leadership characteristics, opportunities, challenges and proposals from a gender perspective. Questionnaires were applied to 55 women from the municipality of Leon, Guanajuato. Results: Some of the leader's characteristics demonstrate: initiative, security, commitment, honesty, leadership, knowledge, character, entrepreneurship, responsibility and sensibility, power of decision, cooperation, strength, confidence, solidarity, autonomy, service and pursuit of the wellbeing. Opportunities for participation

[1] Doctora en Educación. Universidad de Guanajuato, México. luciarg@prodigy.net.mx

[2] Master en Desarrollo Organizacional y Humano. Universidad de Guanajuato, México. l.rodriguez@ugto.mx 
in public life have more demands; however, there is still a lack of gender-inclusive programs. Regarding the difficulties they faced to project their ideas of change, they mentioned as major obstacles the machismo, discrimination and lack of educational opportunities; the housewife's role, the lack of development programs, struggle for the feminine ideals, freedom of speech, lack of initiative and credibility, oppression, violence and abuse. The proposals to improve the options of development have as main approaches: training, education, empowerment, employment, opportunities, government support, fair laws, skills recognition, respect, teamwork and human development. The information is relevant to characterize the successful cases of women who have managed to transcend into leadership positions within a typically conservative society in the Mexican context.

Keywords: Women; leadership; opportunities for participation; Mexican context.

\section{Introducción}

Este documento forma parte de un proyecto más amplio, concluido y realizado en el período de 2011 a 2013, con el propósito de identificar en un grupo de mujeres las características de liderazgo, oportunidades, dificultades y propuestas desde la perspectiva de género. En la sociedad actual, al parecer es cada vez más frecuente que las mujeres tomen decisiones conscientes acerca de sus vidas y se responsabilicen de sus acciones, dando prioridad a cambios que favorecen sus ideales. Sin embargo, las diferencias culturales y el arraigado pensamiento machista han obstaculizado el reconocimiento de los logros de las mujeres, impidiendo así su superación y el acceso a nuevas expectativas.

De acuerdo con los Objetivos de Desarrollo del Milenio (ODM), la igualdad entre los géneros y el empoderamiento, representan elementos vitales para asegurar que las mujeres tengan voz y voto en todas las instituciones, que puedan participar e influir en las decisiones que determinarán el futuro de sus familias y comunidades (ONU, 2012). El Estado mexicano actualmente busca promover la participación política de las mujeres a través de la firma de tratados internacionales.

Respecto a la brecha económica de género, a nivel mundial, México ocupa el lugar número 84 de 135 países evaluados en 2012, habiendo avanzado 5 puestos en la escala con respecto al año anterior (World Economic Forum, 2012). No obstante, la mujer sigue quedándose atrás en lo que respecta a ingresos y productividad, así como al peso que se otorga a su opinión en la sociedad. Ante esta circunstancia se justifica indagar vías que progresivamente conduzcan a la democracia de género, desde ahora y pensando a futuro, el impacto que tendrá en las próximas generaciones, donde será crucial una reducción de las disparidades en los diferentes sectores de la sociedad. 
La falta de programas de promoción para la equidad en puestos de dirección y, por consiguiente, las remuneraciones justas, requieren apoyarse en políticas correctivas de las desigualdades de género persistentes. Algunos antecedentes muestran que a las mujeres se les otorgan menos oportunidades para ocupar puestos de liderazgo en las empresas, aun teniendo preparación y potencial. Del mismo modo se ha identificado en el campo laboral, que se les contrata para desempeñar puestos subordinados o aquellos donde se realizan funciones rutinarias, es decir, al margen de los roles de alta dirección y de toma de decisiones, limitando con esto el acceso a recompensas, promociones y poder. Es decir, menospreciando la capacidad de liderazgo, la igualdad de oportunidades y la credibilidad hacia la población femenina.

En otros países se han implementado legislaciones con el objetivo de garantizar la participación de la mujer. En México, se localizan algunas instancias que ya integraron la perspectiva de género, como el programa: "Hacia la igualdad de género y la sustentabilidad ambiental" (SEMARNAT, 2007), que busca fortalecer los objetivos, ejes, lineamientos y recomendaciones sobre la eliminación de todas las formas de discriminación contra la mujer. Esto representa un avance en el proceso nacional de transversalidad e institucionalización, en tanto propicia la igualdad de oportunidades para las mujeres y los hombres; asimismo la sustentabilidad ambiental en la definición de las políticas públicas.

Así pues, son necesarias más acciones e investigaciones en torno a la democracia de género y, a la vez, superar la brecha entre hombres y mujeres, con el propósito de garantizar bienestar social y una vida con equidad para todas las personas.

\section{Revisión de literatura}

Existe amplio acuerdo de poner a las personas en el centro del desarrollo basado en los pilares fundamentales de: igualdad, sustentabilidad, productividad y empoderamiento. Este último concepto derivado del anglicismo empowerment se aplica como sinónimo de facultamiento y poder de autodeterminación.

El liderazgo se refiere a la fortaleza de carácter, como a la habilidad de formar buenos equipos y las reservas internas de fuerza emocional (Hayward, 2006). Es necesario que el líder mantenga el control de sí mismo e inspire seguridad, ya que debe transmitir confianza en sus propias capacidades, habilidades y fortalezas como persona antes de guiar con éxito a los demás.

Estudios previos confirman que actualmente la población femenina tiene más oportunidad de incorporarse con un papel de liderazgo en los cargos de dirección sin dejar de reconocer que aún persisten pautas y modelos patriarcales, tal como lo menciona Heller (2002). Según Medina (2010), la participación política de las mujeres tiene como líneas de atención la identificación de los principales avances y restricciones 
que impiden la injerencia equilibrada de mujeres y hombres en los espacios de poder público y adopción de decisiones, a fin de garantizar el ejercicio de la ciudadanía en condiciones de equidad.

En un estudio que cuestiona si ¿emplean hombres y mujeres diferentes estilos de liderazgo? se analiza la influencia de tales estilos en el acceso a los puestos de dirección. Cuadrado (2003), enfatiza las diferencias de género y liderazgo, así como la limitación del acceso de las mujeres a la función directiva. De cierto modo, es importante considerar los valores, los estereotipos de género, el uso del poder y las expectativas, ya que son aspectos que no se pueden excluir de los anhelos de las personas y sus aspiraciones, cuando la interacción en grupo es directa y equitativa.

Según esta autora, cabe mencionar que los estilos de liderazgo varían entre mujeres y hombres. El estilo masculino se identifica por la competitividad, la autoridad jerárquica, un alto control y la resolución analítica de problemas, de cierta manera un estilo muy tradicional de relacionarse con los demás. Por otro lado, el estilo femenino se denomina alternativo ya que se representa por la cooperación, la colaboración entre líder y los subordinados, la resolución de problemas basada en la intuición y la empatía.

En cuestión de liderazgo, las mujeres han sido catalogadas de numerosas maneras según estereotipos que adjudican roles asignados socialmente: la de madre abnegada y sumisa; la manipuladora que maneja sus encantos femeninos para que se hagan las cosas a su manera; la adicta al trabajo que no puede delegar responsabilidades; y por último, la igualitaria, que niega el poder de su liderazgo y clama por tratar a los subordinados como colegas.

Heller (2002) comenta una serie de situaciones confusas y contrapuestas en la mayoría de los individuos, siendo un buen momento para reflexionar acerca de los nuevos desafíos que se abren en el tercer milenio, donde mujeres y varones tienen diferentes historias y papeles en la sociedad.

El modelo dominante, que persiste en las organizaciones burocráticas y piramidales en una cultura patriarcal, tiende según Eisler (cit. por Heller, 2002, p.47), a desplazarse a estructuras laborales más planas, integrales y centradas en las personas y los proyectos, dando lugar a modelos donde las mujeres puedan desarrollar mejor sus capacidades y habilidades.

Distintas estudiosas (Chávez, 2004; Heller, 2002 y Zabludovsky, 2002), plantean las particularidades que presentan las mujeres cuando irrumpen en distintos ámbitos públicos (económico, político, social, académico) y los estilos diferenciados de liderazgo que ejercen respecto de sus pares. En este caso, se menciona el estilo de liderazgo alternativo denominado "femenino" (Cuadrado, 2003), que se caracteriza 
por enfatizar la cooperación en lugar de la competición, y la igualdad frente a una jerarquía de superior a subordinado.

Por otra parte, en los últimos años la participación de las mujeres mexicanas en la política se ha visto alentada por las cuotas de género y la paridad democrática, que se sustentan en la igualdad de derechos para ocupar los cargos de representación y espacios de poder al interior de las instituciones.

\section{Materiales y métodos}

En este estudio descriptivo y exploratorio, se aplicaron procedimientos de observación directa para establecer contacto y seleccionar una muestra de participantes en la ciudad de León, Guanajuato, México.

Posteriormente se llevó a cabo un trabajo de campo mediante la administración de instrumentos tipo encuesta aplicados a un grupo de 55 mujeres, entre las que se ubican líderes políticas, emprendedoras y representantes de asociaciones, que corresponden a los grupos seleccionados en esta investigación.

Con los datos recabados se procedió al análisis y procesamiento de la información mediante base de datos que concentran la captura de los cuestionarios y la consiguiente interpretación cualitativa de los mismos.

\section{Resultados y discusión}

Los hallazgos confirman una creciente participación de las mujeres en los espacios públicos extra domésticos. De igual forma, en las áreas urbanas de León, Guanajuato, resulta factible identificar una creciente proporción de emprendedoras y jefas de pequeñas y medianas empresas, así como ejecutivas de corporaciones instaladas en el corredor industrial. En este caso, se aportan datos de 55 personas representativas de diferentes ámbitos: político empresarial y social, a fin de obtener una visión más amplia de las categorías estudiadas.

Los principales rasgos aludidos en torno al liderazgo de las mujeres pudieran resumirse como sigue: Iniciativa, poder de decisión, compromiso, capacidad, sensibilidad, cooperación, inteligencia y valores de honestidad, fortaleza, confianza, solidaridad, autonomía, servicio, búsqueda del bien común y actitud emprendedora.

Las oportunidades que actualmente tienen las mujeres de participación en la vida pública han aumentado porque se han abierto varias opciones en cuanto a la ideología, la legislación, e incluso la posibilidad de aspirar a gobernar un país; pero todavía persisten dificultades para que proyecten sus ideas de cambio por prejuicios fuertemente arraigados en los contextos diversos. 
Algunas de las voces recuperadas en las mujeres del presente estudio manifiestan las dificultades que viven cotidianamente:

- "Desafortunadamente nuestra cultura "machista" continúa siendo una limitante, aunque se ve mayor cantidad de mujeres económicamente productivas y líderes. No hay igualdad de condiciones".

_"Represión social por parte de los hombres y falta de confianza en la mujer".

_"El principal obstáculo es que por el simple hecho de ser mujer, la mayoría de las veces ni siquiera escuchan sus propuestas".

_ "Actualmente existe una brecha de género en la sociedad, que implica que la mayoría de las situaciones y actuar de las personas sean o tengan una mirada masculina, dejando a un lado las opiniones y expresiones de las mujeres. Los estereotipos, roles de género y todo lo que implica en si la palabra género, provoca una cultura excluyente y no incluyente; por lo que las mujeres son etiquetadas en un ambiente no reconocido ni valorado..."

Las propuestas enunciadas por las personas consultadas indican capacitación, equidad de sueldos, leyes, credibilidad y reconocimiento a las mujeres emprendedoras.

- "Crear administraciones incluyentes (...), que brinden las mismas oportunidades de crecimiento y desarrollo a mujeres y hombres; impulsar y crear políticas públicas encaminadas al reconocimiento, valoración y ejercicio pleno de los derechos de las mujeres; crear ambientes laborales de acuerdo a las necesidades de las mujeres y hacer valer en cuanto a la remuneración, el mismo sueldo para mujeres y hombres, por el mismo trabajo..."

- "Educar a los hijos fomentando la igualdad de género. Ellos son el futuro..., si desde sus cimientos educativos obtienen valores familiares de igualdad de género, cambiaremos al país".

- "Revisar los modelos educativos que incluyan perspectiva de género; dar más poder a las instancias que trabajan en políticas de la mujer y así poder incidir transversalmente en los medios de comunicación, las leyes, los programas, el trabajo, etc."

- "Legislar para que haya el mismo número de hombres y mujeres en los cargos públicos".

- "Impulsar el desarrollo profesional, laboral y personal de las mujeres". 
- "Crear programas de inclusión, elaborar talleres de liderazgo político y organizacional”.

- "Igualdad de salarios; que no haya distinción en puestos líderes".

En suma, las expresiones anteriores aportan visiones que hacen pensar en un cambio de conciencia y actitudes respecto a una perspectiva renovada del papel que juegan las mujeres. Helgesen, Rosener y Kaufmann (cit., por Cuadrado, 2003), resaltan el modo en que las mujeres líderes trabajan y se comunican de manera más receptiva y participativa, caracterizado esto por la cooperación y la equidad, e incluso comparten el poder y la información. De igual forma otorgan especial importancia a la parte emocional y al sentido humano, pues realzan el trabajo de los demás y responden de forma más adecuada a las necesidades organizativas y de colaboración a lo largo del proceso.

Hay maneras diferentes de definir qué es un líder, aunque uno de los estilos que más se adapta al trabajo de las mujeres pudiera ser el liderazgo participativo, ya que permite que los colaboradores tomen decisiones importantes dentro del grupo. Lo anterior se puede observar a nivel local en instituciones dirigidas por mujeres, quienes tienen mayor sensibilidad hacia problemas de tipo social y, por lo mismo, emprenden acciones de liderazgo en asociaciones y voluntariados sin fines de lucro, con la meta de beneficiar a los sectores más vulnerables de la población

En este sentido, pudiera adoptarse la dimensión estratégica de fortalecimiento, que integrada con otras acciones de mayor alcance a favor de las mujeres, promueva un impacto trasversal en las esferas políticas y en la cultura organizacional, donde con frecuencia se perciben los desequilibrios de género desde lejos y como parte de un patrimonio cultural inamovible y persistente.

\section{Conclusiones}

A pesar de la creciente incorporación al mercado del trabajo, falta incluir efectivamente la perspectiva de género y promover la participación igualitaria de hombres y mujeres, tanto al interior de las familias y en todos los niveles de toma de decisiones.

Desde la política pública se tiene el ámbito idóneo para hacer visibles las desigualdades en el uso del poder, a fin de impulsar relaciones más horizontales, con empoderamiento suficiente para que todas las personas puedan aprovechar las oportunidades y los beneficios de la democracia en las esferas de la vida pública y privada. Las mujeres guanajuatenses encuestadas relataron las oportunidades que tienen para participar en el desarrollo local y regional. 
En el estudio se consideró importante identificar las condiciones y dificultades inherentes al liderazgo, así como las necesidades y áreas de oportunidad a las que pueden aspirar las ciudadanas desde los enfoques de la democracia y equidad de género. Además, sería interesante llevar a cabo investigaciones que comparen la situación de las mujeres mexicanas con las de otros países latinoamericanos.

Por tanto, el proyecto realizado abona información relevante para caracterizar los casos exitosos de mujeres que han logrado trascender hacia puestos de liderazgo dentro de una de sociedad típicamente conservadora al interior del contexto mexicano.

Los hallazgos, ofrecen una perspectiva prometedora de un estudio repensado bajo los aspectos de la igualdad de género para el desarrollo de las mujeres y sus familias. Se sugiere dar continuidad al presente trabajo e iniciar otros estudios que aborden investigaciones desde un enfoque de género y empoderamiento de las mujeres.

\section{Lista de referencia}

CUADRADO, Isabel (2003). ¿Emplean hombres y mujeres diferentes estilos de liderazgo? Análisis de la influencia de los estilos de liderazgo en el acceso a los puestos de dirección. Revista de Psicología Social. Madrid. 18(3) pp. 283-307.

CHÁVEZ, Julia del Carmen (2004). Perspectiva de género. Plaza y Valdés, México.

HAYWARD, Sue (2006). Liderazgo femenino. Ed. Continental, México.

HELLER, Lidia (2002). Nuevos Estilos de Liderazgo femenino en organizaciones. ¿De dónde venimos y hacia dónde vamos? En: Gina Zabludovsky, Mujeres en Cargos de Dirección en América Latina. Estudios sobre Argentina, Chile, México y Venezuela. UNAM, Porrúa, México.

HELLER, Lidia (2011). Mujeres en la cumbre corporativa: el caso de la Argentina. Revista del Centro de Estudios de Sociología del Trabajo. No. 3, pp. 68-96, Universidad de Buenos Aires.

MEDINA, Adriana (2010). La participación política de las mujeres. De las cuotas de género a la paridad. CEAMEG, México.

MEENTZEN, Angela y Enrique Gomáriz (2003). La democracia de género en el marco de las estrategias de acción. En: Democracia de Género, una propuesta inclusiva. Contribuciones desde América Latina y Europa. Fundación H. Böll, México.

ORGANIZACIÓN DE LAS NACIONES UNIDAS (2012). Objetivos de Desarrollo del Milenio: Informe 2012. ONU. 
SEMARNAT (2007). Programa Hacia la igualdad de género y la sustentabilidad ambiental 2007-2012. Secretaria de Medio Ambiente y Recursos Naturales, México.

WORLD ECONOMIC FORUM (2012). The Global Gender Gap Report 2012, USA.

ZABLUDOVSKY, Gina (2002). Mujeres en Cargos de Dirección en América Latina UNAM. Porrúa, México. 